\title{
Total Intestinal Aganglionosis
}

National Cancer Institute

\section{Source}

National Cancer Institute. Total Intestinal Aganglionosis. NCI Thesaurus. Code C101074.

A complete lack of ganglia in the intestine. This is an extremely severe form of

Hirschsprung Disease. 